\title{
Issues of quality in providing services for subfertile couples
}

F Song, J M Mason, S Ibbotson, N Freemantle, A F Long, T A Sheldon

This review is based on Effective Health Care, Bulletin No 3

The effectiveness of subfertility treatments has recently been reviewed in Effective Health Care. ${ }^{1}$ Many health authorities are coming under increasing pressure to purchase treatments for subfertile couples, though because treatments are perceived to be ineffective ${ }^{2}$ few districts currently purchase comprehensive services. The review in Effective Health Care found that many treatments that include assisted conception (such as in vitro fertilisation - embryo transfer (IVF-ET)) are effective, given appropriate indications for their use (table). ${ }^{1}$ The decision whether to purchase these services or how much to purchase is not just a technical question and will need to be located within the contexts of general fertility services within a district and of competing claims on resources.

However, since purchasing in this area will probably increase, this paper examines some of the key questions which will need to be addressed to ensure high quality service provision.

\section{Size of problem}

A health authority with an established subfertility service and a population of 250000 , with 46000 women aged $20-44$ years, will probably receive around $230(0 \cdot 5 \%)$ new consultant referrals each year. ${ }^{3}$ This is higher than the historical annual met demand of about $0.37 \%$ a year ${ }^{4}$ and may increase owing to demographic changes and raised public awareness of treatment possibilities. ${ }^{3}$

Subfertility can cause considerable psychosocial distress affecting many areas of a couple's life. ${ }^{5}$ It may result in social handicap, preventing fulfilment of social roles and realisation of personal and societal expectations for parenthood. ${ }^{4}$ For infertile couples "biological parenthood seems to be a crucial factor in their sense of control and fulfilment in their lives." 8

\section{Quality of evidence}

Because many treatments have not been evaluated by randomised controlled trials the major evidence for effectiveness must be based on the outcomes recorded from large retrospective case series and routine data compared with what is known of the outcomes for untreated patients. This evidence is less reliable than that produced by a well designed randomised controlled trial, a controlled trial without randomisation, and a cohort or casecontrol study. ${ }^{9}$ However, when the results of large retrospective series are substantially consistent between centres, over time, and across countries these data may be used to give a broad indication of effectiveness, though it will be subject to biases.

The table summarises the results of the review of effectiveness of subfertility treatments, and details of the evidence for each cause is contained in the bulletin. ${ }^{1}$

\section{Audit of subfertility}

All centres undertaking treatments involving embryos (for example, IVF-ET) or donated gametes (for example, donor insemination) are licensed and subjected to a process of scrutiny and data collection which is published regularly in an aggregated form. ${ }^{1011}$ If extended to all infertility treatments this could develop into a system of external audit. It is essential that audit is carried out with scientific rigour. ${ }^{12}{ }^{13}$ To ensure this several issues have to be taken into account: outcome measures, patient selection, spontaneous conception, and methods of presenting and analysing results.

OUTCOME MEASURES

Ideally the management of subfertility should be evaluated in terms of its success in reducing the psychosocial distress or social handicap due to subfertility. For example, this could include measures of the extent to which interventions help couples come to terms with their childlessness. ${ }^{11}$ However, this is difficult and there is a need to develop valid and standardised measures of quality of life for research in this area.

Reproductive outcomes - Most published reports concentrate on reproductive outcomes $^{14}$ such as biochemical (evidence of) pregnancy, clinical pregnancy, birth rate, and maternity rate. This makes comparison between trials, centres, clinicians, and treatments problematic. Uniformity of outcome measures is required for audit to have any validity. The most useful reproductive outcome for planners and 
Consequences of subfertility treatments

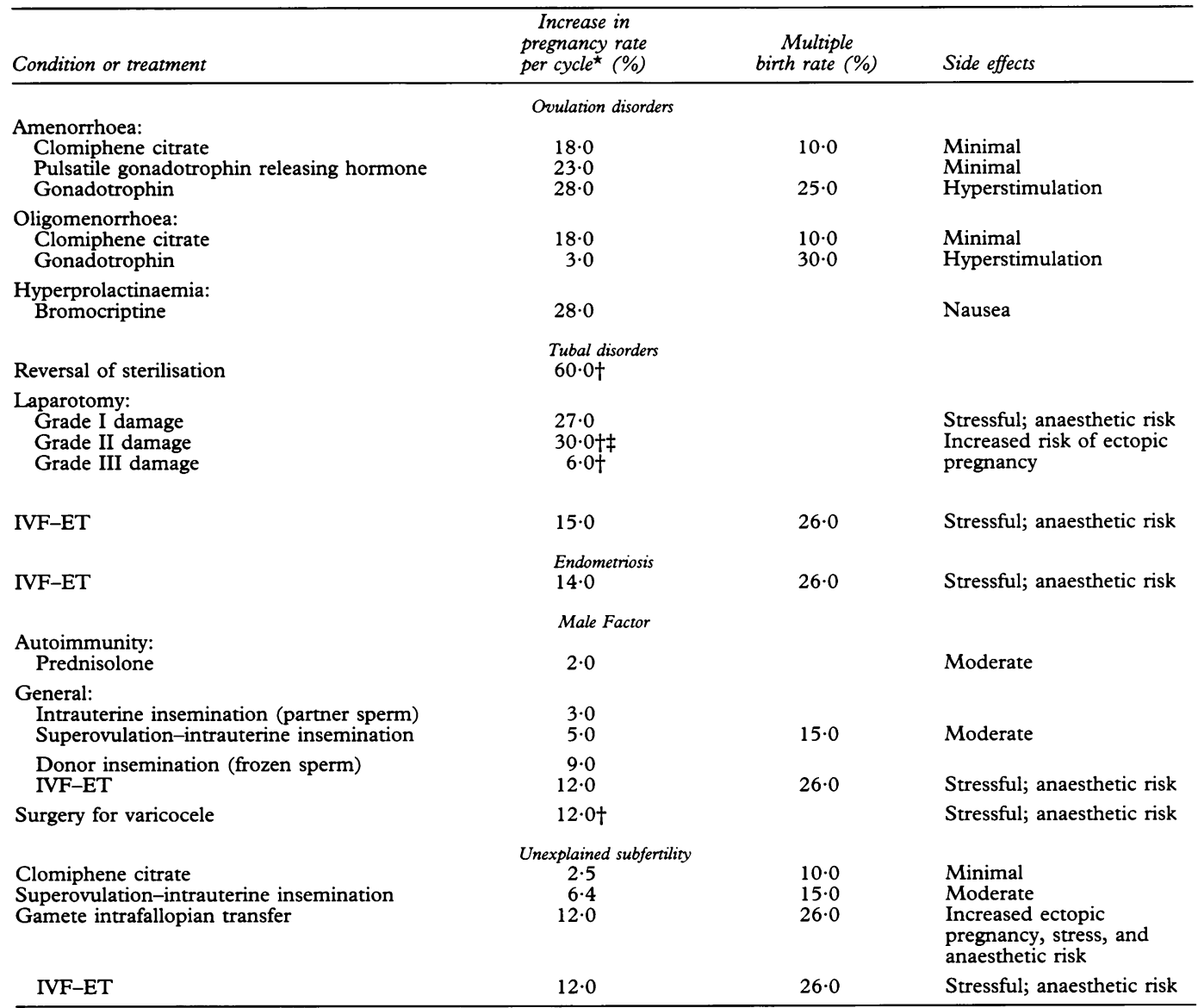

*Pregnancy rate in excess of spontaneous pregnancy: for cyclical treatments these apply to the first few cycles of treatment but then diminish. Reducing these figures by $25 \%$ gives a rough estimate of the maternity rate/cycle.

tCumulative pregnancy rate after two years.

¥The added benefit of surgery for grade II damage seems higher than for grade I damage because of the higher spontaneous pregnancy rate in mild cases.

IVF-ET = in vitro fertilisation - embryo transfer.

consumers is the maternity rate, which is clearly understandable to couples seeking treatment. Reporting the live birth rate overstates success because of the raised incidence of multiple births with some treatments. Other outcomes, such as conception rates, give even more optimistic estimates of success but may indicate where in the treatment cycle there are problems and so can be useful as part of audit (see fig 1). ${ }^{15}$

\section{PATIENT SELECTION}

The reproductive success rate also depends on patient selection. By selecting couples with a very good prognosis (for example, women under 40 years; men with normal sperm) centres can achieve higher rates. For example, some "good" centres report cumulative pregnancy rates from around $35-50 \%$ after three cycles of in vitro fertilisation (IVF). ${ }^{16}{ }^{17}$ The higher rate is partly achieved by selecting only couples who have proved the ability to fertilise by means of a trial of IVF. Higher rates can also be achieved by the selective drop out from treatment of those patients with poorer prognosis. These biases mean that pregnancy rates reported by different centres may not provide a fair basis for comparison. ${ }^{18}$

Similarly, comparison of success rates for tubal surgery must first be standardised for the nature and severity of the tubal disease since surgery is relatively ineffective for severely damaged fallopian tubes.

Characteristics of patients such as age, parity, cause and duration of subfertility, and severity of disease must be clearly stated as centres or treatments may appear more successful because of variations in diagnosis and patient selection. Since purchasers and patients are interested in estimates of the increase in maternity rate attributable to treatment, the maternity rates of those on the waiting list could possibly be used as a baseline, where they exist.

\section{SPONTANEOUS PREGNANCIES}

A significant number of pregnancies will occur without treatment. ${ }^{19} 20$ Effectiveness should be assessed on the basis of the maternity or pregnancy rate over and above these spontaneous pregnancies, otherwise the impact of treatments will be overestimated.

ANALYSIS OF RESULTS

Because the total (cumulative) chance of pregnancy increases with time and number of treatment cycles reports should include the time taken to conceive or the number of treatment cycles, or both. This information can be usefully presented in the form of cumulative conception or maternity curves 


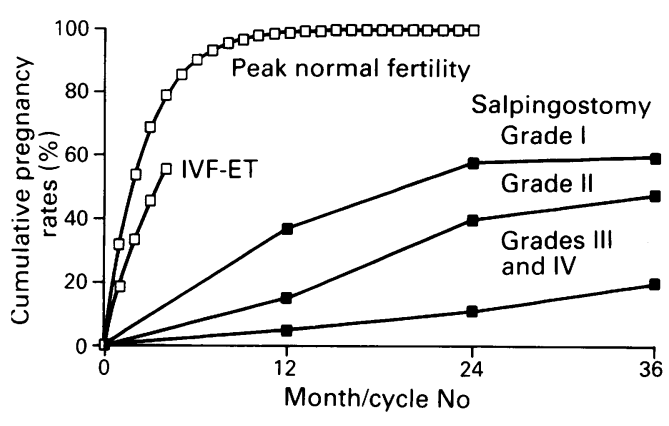

Source: Effective Health Care, Bulletin No 3, 1992

Fig 1 Cumulative pregnancy rates in patients with tubal damage

(for example, as in fig 1). Life table or survival analysis is being used increasingly and can be a powerful means of comparison ${ }^{21} 22$ but requires comprehensive follow up subjects in the original cohort or correct adjustment for those who drop out, in order to avoid biased estimates of effectiveness.

To be able to judge which centres have better reproductive success rates purchasers or patients should seek clear information on outcomes, suitably adjusted for length of follow up, and on the number of treatment cycles and patient selection.

Figure 2 summarises the average reproductive experience of people of receiving IVF and indicates the stages of the treatment process which audit could usefully examine to improve the success of treatments.

\section{Quality issues in services for subfertile couples}

As treatments for subfertility are truly elective, decisions made by couples about whether or not to proceed with particular interventions must be made when they have the relevant information about personal costs and acceptability and likely outcomes. As couples often go to considerable lengths and personal expense in pursuing treatment this poses a particular responsibility on those providing such treatments. ${ }^{23}$

Many couples feel the need for information, support, and other forms of counselling, especially around the time of diagnosis. ${ }^{24}$ There is evidence that stress is reduced in couples who feel involved in and in control of their treatment. ${ }^{25}$ There has been little systematic research into the effectiveness of counselling for couples seeking treatment for subfertility; several models of support have been used. ${ }^{6}{ }^{26-28}$ Further research is needed, including ways of better identifying those who are particularly vulnerable and likely to benefit. ${ }^{29}$

It is important to acknowledge the social context of reproductive technologies, in particular the way they may reinforce stereotypic attitudes to women and motherhood. There is the risk that the increased availability of more effective treatments may result in childless women coming under greater pressure to reproduce. ${ }^{30}$ Therefore providing independent information and support are central components of a high

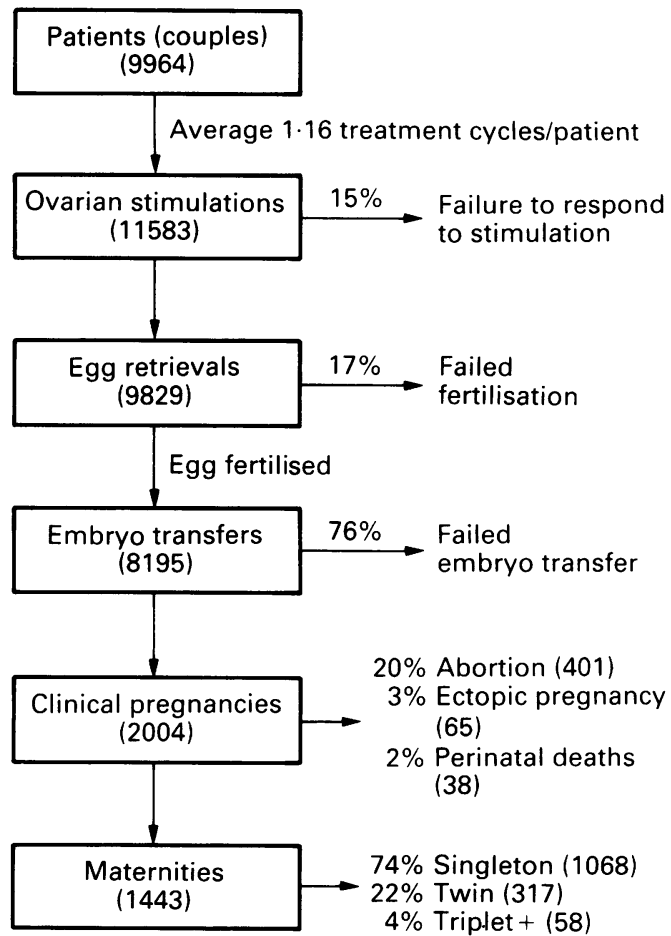

Fig 2 Average reproductive experience of patients receiving in vitro fertilisation-embryo transfer (IVF-ET) (data for United Kingdom, 1990) ${ }^{11}$

quality service. It is also important that nonreproductive outcomes feature in the audit of a service for subfertile couples.

\section{Organisational issues}

If an authority decides to purchase subfertility services several organisational issues need to be considered. There is a strong case for subfertility services to be managed predominantly in specialised tertiary units for the following reasons.

(1) The management of subfertility is a complex task requiring skills which are not necessarily available in a district general hospital.

(2) These skills are more likely to be maintained where there is a sizable volume of activity. Routinely collected data in the United Kingdom indicate that, generally, treatment centres with high levels of activity have better success rates than smaller ones. ${ }^{11}$

(3) If subfertility services are carried out at district general hospitals without specialist facilities treatments may be offered according to their availability rather than their appropriateness. For example, the surgical treatment of tubal damage results in an increase in cumulative pregnancy rate after two years of nearly $30 \%$ for mild and moderate cases but only $6 \%$ for severe cases. ${ }^{1}$ Treatment with IVF techniques for women with more severe disease would be more appropriate, ${ }^{31}$ yielding an average pregnancy rate per cycle of $18 \% .^{11}$

(4) Because of economies of scale it is unlikely that a district centre could operate efficiently. For example, the most efficient treatment capacity for an IVF-ET clinic has been estimated at around 750 started 
treatments each year (G Haan, WHO Europe conference on the place of in vitro fertilisation in infertility care, Copenhagen, 1990). ${ }^{32}$ Large centres in the United

Kingdom saw on average 650 patients for assisted conception in $1990 .^{11}$

For patients to receive the most effective management and for resources to be used most efficiently districts wishing to purchase these services should have direct access to a comprehensive range of diagnostic and treatment services so that appropriate choices can be made. However, to avoid unnecessary duplication of diagnostic testing and treatment and to reduce the stress experienced by patients it is important that all providers of services to a given population agree on and adhere to a common management philosophy and framework. This is likely to optimise the continuity of care for individuals as they move between primary, secondary, and tertiary levels of care.

To help this process, protocols for managing subfertility for a district or family health services authority population should be agreed with the tertiary centres with which contracts are placed. These protocols should address the roles of primary and secondary care in early management. Therefore clear guidelines are required about appropriate criteria for the composition of the initial work up and referral for use in primary care as treating couples too early leads to an inefficient use of resources due to the high spontaneous pregnancy rate. A recent survey has indicated that general practitioners would welcome this (J Kurinczuk, M Clarke, University of Leicester; personal communication). The recent guidelines of the Royal College of Obstetricians and Gynaecologists and those developed at the University of Aberdeen (A Templeton, personal communication) might possibly form the basis of such guidelines. These would be valuable in helping to promote a more homogeneous and effective approach in practice and should be incorporated into services specifications.

Given the enthusiasm of providers and the readiness of some couples to undergo many treatment cycles and any procedures offered, districts that do decide to purchase subfertility services need to keep tight contractual control over the volume of activity and the quality of care. Management guidelines are of central importance to achieving a cost effective strategy.

For acknowledgements see reference 1 .

1 School of Public Health, University of Leeds; Centre for Health Economics, University of York; Research Unit, Royal College of Physicians. The management of subfertility. Leeds: University of Leeds, 1992. (Effective Health Care, bulletin No 3.)
2 Harrison S, Wistow G. The purchaser/provider split in English health care: towards explicit rationing? Policy and Politics 1992;20:123-30.

3 Hull MGR, Glazener CMA, Kelly NJ, Conway DI, Foster PA, Hinton RA, et al. Population study of the causes, treatment and outcome of infertility. BMF 1985;291:1693-7.

4 Page $\mathrm{H}$. In vitro fertilisation and the NHS - planning issues in in vitro fertilisation [PhD Thesis]. Leeds: University of Leeds, 1992. 200pp

5 Wright S, Allard M, Lecours A, Sabourin S. Psychosocial distress and infertility: a review of controlled research. Int f Fertil 1989;34:126-42.

6 Freeman EW, Boxer AS, Rickels K, Tureck R, Mastroianni L. Psychological evaluation and support in a programme of in vitro fertilisation and embryo transfer. Fertil Steril 1985;43:48-53.

7 Taymor ML, Bresnick E. Infertility counselling. In: Taymor ML, ed. Infertility. New York: Grune and Stratton, 1978:94.

8 Strickler J. The new reproductive technology: a problem or solution. Sociology of Health and Illness 1992; 14:111-32.

9 Long AF, Sheldon TA. Enhancing effective and acceptable purchaser and provider decisions: overview and methods. Quality in Health Care 1992;1:74-6.

10 Interim Licensing Authority. The sixth report of the Interim Licensing Authority for human in vitro fertilisation and embryology, 1991. London: Interim Licensing Authority, 1991.

11 Human Fertilisation Embryology Authority. Annual report. London Human Fertilisation Embryology Authority, 1992.

12 Russell IT, Wilson BJ. Audit: the third clinical science? Quality in Health Care 1992;1:51-5.

13 Mugford $M$, Banfield P, O'Hanlon $M$. Effects of feedback information on clinical practice: a review. $B M \mathcal{F}$ 1991;303:398-42.

14 Ryan M, Twaddle S. Infertility: an overview. Aberdeen: Health Economics Research Unit, University of Health Economics Research Unit, Universit

15 Page $H$. Calculating the effectiveness of in-vitro Page $\mathrm{H}$. Calculating the effectiveness of in-vitro
fertilisation. A review. $\mathrm{Br} f$ Obstet Gynaecol 1989;96:334-9.

16 Tan SL, Royston P, Campbell S, Jacobs HS, Betts J, Mason B, et al. Cumulative conception and livebirth rates after in-vitro fertilisation. Lancet 1992;339:1390-4.

17 Hull MGR, Eddowes HA, Fahy U, Abuzeid MI, Mills MS, Cahill DJ, et al. Expectations of assisted conception for infertility. BMf 1992;304:1465-9.

18 Hershlag A, Kaplan EH, Loy RA, DeCherney AH, Lavy G. Selection bias in in vitro fertilization programs. $A m \mathcal{F}$ Obstet Gynecol 1992;166:1-3.

19 Collins JA, Wrixon W, Janes LB, Wilson EH. Treatmentindependent pregnancy among infertile couples. New Engl f Med 1983;309:1201-5.

20 Lilford RJ, Dalton ME. Effectiveness of treatment for infertility. $B M Y$ 1987;295:155-6.

21 Cooke ID, Salaiman RA, Lenton EA, Parsons RJ. Fertility and infertility statistics: their importance and application. Clinics in Obstetrics and Gynaecology 1981;8:3.

22 Cramer DW, Walker AM, Schiff I. Statistical methods in evaluating the outcome of infertility therapy. Fertil Steril 1979;32:80-6.

23 Leiblum SR, Kemmann E, Lane MK. The psychologic concomitants of in vitro fertilisation. fournal of Psychosomatic Obstetrics and Gynaecology 1987;6:165.

24 Daniluk J. Infertility: intrapersonal and interpersonal impact. Fertil Steril 1988;49:982-90.

25 Abbey A, Halman LJ, Andrews FM. Psychosocial, treatment, and demographic predictors of stress associated with infertility. Fertil Steril 1992;57:122-8.

26 Rosenfeld DL, Mitchell E. Treating the emotional aspects of infertility: counselling services in an infertility clinic. Am ₹ Obstet Gynecol 1979;135:177.

27 Sarrell PM, DeCherney AH. Psychotherapeutic intervention for treatment of couples with secondary intervention for treatment of coup

28 Berger DM. The role of the psychiatrist in a reproductive biology clinic. Fertil Steril 1977;28:141-5.

29 Connolly KJ, Edelmann RJ, Cooke ID, Lenton E. Investigation of counselling needs and outcomes for IVF patients: report to the Department of Health. 1992.

30 Sheldon TA, Ibbotson S. Public health aspects of subfertility. In: Drife JO, Templeton A, eds. Infertility. London: Springer-Verlag, 1992.

31 Lilford RJ, Watson AJ. Commentary. Has in-vitro fertilization made salpingostomy obsolete? $\mathrm{Br} \mathcal{F}$ Obstet Gynaecol 1990;97:557-60.

32 Stephenson PA, Svensson PG. In vitro fertilisation and infertility care: a primer for health planners. International fournal of Health Sciences 1991;2:119-23.

33 Royal College of Obstetricians and Gynaecologists Fertility Subcommittee. Infertility: guidelines for practice. London: RCOG, 1992. 